(C) 1998 International Press

Adv. Theor. Math. Phys. 2 (1998) 293-302

\title{
S-Duality and Global Symmetries
}

\author{
Philip C. Argyres ${ }^{1}$ \\ Newman Laboratory \\ Cornell University \\ Ithaca, NY 14853-5001, U. S. A.
}

\begin{abstract}
S-dualities in scale invariant $N=2$ supersymmetric field theories are derived by embedding those theories in asymptotically free $N=2$ theories with higher rank gauge groups. S-duality transformations on the coupling of the scale invariant theory follow from global symmetries acting on the Coulomb branch of the higher rank theory. Since these global symmetries are exact in the asymptotically free theory, this shows that S-duality is an exact equivalence of $N=2$ theories and not just a property of their supersymmetric states.
\end{abstract}

\section{Introduction}

S-duality refers to a quantum equivalence of classically inequivalent field theories. This equivalence often consists of the identification of a theory with the same theory deformed by an exactly marginal operator. These deformations can be viewed as transformations on the classical space of couplings of the theory, and the duality group is the group of such transformations. Since the elements of the duality group are supposed to connect equivalent theories, the quantum coupling space is the classical one divided by the action of the duality group. The paradigmatic example [1] is the strong-weak coupling duality of $N=4$ supersymmetric Yang-Mills theory under which theories with couplings $\tau$ and $-1 / \tau$ are identified. Since the $N=4$ theory

\footnotetext{
${ }^{1}$ argyres@mail.lns.cornell.edu
} 
is scale invariant the exactly marginal operator corresponds to changing the coupling.

This letter discusses related S-dualities for scale invariant $N=2$ supersymmetric field theories. Up to now, the evidence for S-dualities in field theory has come from the spectrum of BPS saturated states [2] and from low-energy effective actions [3-4]. This evidence leaves open the possibility that the non-BPS saturated spectrum and scattering amplitudes at finite momenta are not left invariant under S-duality transformations; $c f$. [5]. By relating S-duality transformations in scale invariant $N=2$ field theories to global symmetries in asymptotically free theories, I will show that these S-dualities are, in fact, exact equivalences of their quantum field theories.

The basic idea is to regard the marginal couplings of the scale invariant theory as lowest components of vector multiplets-complex scalar "Higgs" fields - in an enlarged theory. Then the coupling space $\mathcal{M}$ of the scale invariant theory is realized as a submanifold of the Coulomb branch $\mathcal{C}$ of the enlarged theory. Any S-duality identifications of different points of $\mathcal{M}$ are interpreted as equivalences on $\mathcal{C}$. By choosing the enlarged theory appropriately, these equivalences on $\mathcal{C}$ can be made manifest as (spontaneously broken) global symmetries.

Some elementary properties of the enlarged theory can be deduced immediately. The couplings of the enlarged theory must not get strong in the ultraviolet, for then we would have no control over the Coulomb branch for large Higgs vevs since it would depend on whatever physics regulates the theory at short distances. Furthermore, if the enlarged theory is itself scale invariant, then the couplings of the smaller scale invariant theory of interest will typically depend on the couplings (as well as the Higgs vevs) of the enlarged theory. One can find in this way relations among S-duality groups for different theories $[6,7]$, but not the kind of derivation of S-duality that we are looking for. Thus we should look for asymptotically free enlarged theories for which we have exact information about the Coulomb branch for all values of the Higgs vevs.

In preparation for making the argument outlined above more concrete, I first briefly describe the (conjectured) S-duality groups of the theories I will use as examples, and then review the exact solutions for the Coulomb branches of these models. However, it should be clear that this line of argument can be applied to a much wider set of models than the examples I treat below. In particular, it would be interesting to extend it to the series with $S p(2 r)$ gauge group, one antisymmetric and four fundamental hypermultiplets [4], as well as to the rich set of recently solved models with product gauge groups [8]. Also, although one cannot derive the S-duality group of $N=4$ theories in this way from enlarged theories with simple gauge groups, it may be possible to do so using enlarged theories with product 
gauge groups. Note, in this connection, that there is no requirement that the enlarged theory have as much supersymmetry as the scale invariant theory; in particular, one can flow from an $N=2$ supersymmetric theory to an $N=4$ one in the infrared.

\section{Some $N=2$ S-dualities.}

In the $N=2$ theories discussed here it is convenient to define the coupling as $\tau=\frac{\vartheta}{\pi}+i \frac{8 \pi}{g^{2}}$, differing by a factor of two from the usual definition. The $N=2$ theory with $S U(2) \simeq S p(2)$ gauge group and four fundamental "quark" hypermultiplets has S-duality group ${ }^{2} S L(2, \mathbb{Z})$ [9]. The classical coupling space $\mathcal{M}_{c l}=\{\operatorname{Im} \tau>0\}$ is identified under the transformations $T: \tau \rightarrow \tau+1$ and $S: \tau \rightarrow-1 / \tau$, giving the quantum coupling space $\mathcal{M}=\mathcal{M}_{c l} / S L(2, \mathbb{Z})$. The $S L(2, \mathbb{Z})$ duality group can be presented abstractly as the group with two generators $S$ and $T$ satisfying the relations $S^{2}=(S T)^{3}=1$. The generators $S$ and $S T$ do not act freely on $\mathcal{M}_{c l}$, but have fixed points which are $\mathbb{Z}_{2}$ and $\mathbb{Z}_{3}$ orbifold points of $\mathcal{M}$, if we assign $\mathcal{M}_{c l}$ a flat metric. The relations satisfied by the generators encode the holonomy in $\mathcal{M}$ around these orbifold points.

Since we are free to make coordinate changes on $\mathcal{M}_{c l}$, though the topology of $\mathcal{M}$ certainly has an invariant meaning, it may not be clear that the holonomies around points in $\mathcal{M}$ are physically meaningful. In particular, to define holonomies, one needs a connection. The existence of a natural connection on coupling space [10] implies that these holonomies are physical [11]. Thus the S-duality group, considered as an abstract infinite discrete group is physically meaningful.

The scale invariant $N=2$ theories with a single $S U, S O$, or $S p$ gauge group and quarks in the fundamental (defining) representation all have lowenergy effective theories that are invariant under identifications of $\tau$ under a discrete group isomorphic to $\Gamma_{0}(2) \subset S L(2, \mathbb{Z})[6,7] . \Gamma_{0}(2)$ is the subset of $S L(2, \mathbb{Z})$ matrices with even lower off-diagonal entry, or equivalently, is the subgroup of $S L(2, \mathbb{Z})$ generated by $T$ and $U=S T^{2} S T$. The only relation satisfied by these generators is $U^{2}=1$, thus characterizing $\Gamma_{0}(2)$ more abstractly as the group freely generated by $T$ and $U$ subject to the one relation $U^{2}=1$. Quotienting the classical coupling space by the S-duality group, we expect a single $\mathbb{Z}_{2}$ orbifold point in the quantum coupling space at the fixed point of the $U$ transformation; see fig. 1. An important feature of this duality group is that it does not remove all the ultra-strong coupling points (the cusp at $\tau=0$ in the figure) from the quantum coupling space.

\footnotetext{
${ }^{2}$ I will only discuss the S-duality action on marginal couplings and not on masses or other operators, and so will ignore the distinction between $S L(2, \mathbb{Z})$ and $P S L(2, \mathbb{Z})$ in what follows.
} 
No weakly coupled description of the ultra-strong coupling points is known for the higher rank theories.

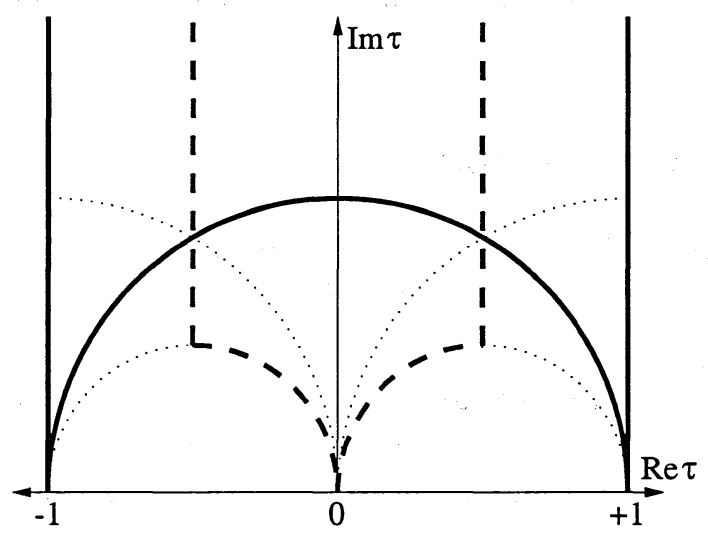

Figure 1: Fundamental domains in the $\tau$ plane of $\Gamma_{0}(2)$ (thick dashed lines) and $\widetilde{\Gamma}^{0}(2)$ (thick solid lines). Weak coupling is at $\tau=+i \infty$ and the ultrastrong coupling points are at $\tau=\{0, \pm 1\}$. The domains are "folded" along the $\operatorname{Im} \tau$ axis and opposite edges are identified, giving rise to $\mathbb{Z}_{2}$ orbifold points at $\tau=\left\{i, \frac{1}{2}(1 \pm i)\right\}$.

In fact, in the form of the solution for the effective action on the Coulomb branch that I use below, only the $S p(2 n)$ series has its S-duality group realized in precisely this way. The $S U(n)$ and $S O(n)$ series realize the duality group instead as the subgroup of $S L(2, \mathbb{Z})$ generated by $S$ and $V=T^{2}$ which I will denote by $\widetilde{\Gamma}^{0}(2)$. (It is related to $\Gamma^{0}(2)$, the subset of $S L(2, \mathbb{Z})$ matrices with even upper off-diagonal entry by a similarity transformation: $\widetilde{\Gamma}^{0}(2)=T \cdot \Gamma^{0}(2) \cdot T^{-1}$.) Its fundamental domain in the $\tau$ plane is also shown in fig. 1. Abstractly it is the freely generated group on $V$ and $S$ with the one relation $S^{2}=1$, and so is isomorphic to $\Gamma_{0}(2)$. Nevertheless, the fact that the S-duality group is realized in these two different ways will be important below in deriving the full $S L(2, \mathbb{Z})$ duality of the $S U(2) \simeq S p(2)$ theory.

\section{Some Exact Solutions for Coulomb Branches}

I refer to the complex adjoint scalar component $\phi$ of vector multiplets as Higgs fields and the two complex scalar components $q, \widetilde{q}$ of hypermultiplets as quarks. The Coulomb branch $\mathcal{C}$ is the manifold of degenerate vacua with zero quark vevs and Higgs vev in the complexified Cartan subalgebra of the gauge group, breaking $G \rightarrow U(1)^{r}$ where $r=\operatorname{rank}(G)$. Locally $\mathcal{C} \simeq \mathbb{C}^{r}$, and I denote its coordinates by $\phi_{a}, a=1, \ldots, r$. Note that the point $\phi_{a}=0$ will be the scale invariant vacuum, but that moving away from it along 
the Coulomb branch to points with $\phi_{a} \neq 0$ spontaneously breaks the scale invariance of the underlying theory and generically flows to the infrared free $U(1)^{r}$ Maxwell theory.

The couplings $\tau_{a b}$ of this low-energy effective $U(1)^{r}$ theory can receive quantum corrections. The low-energy $U(1)^{r}$ theory has an $S p(2 r, \mathbb{Z})$ electricmagnetic duality, implying [12] that the effective couplings $\tau_{a b}$ are a section of an $S p(2 r, \mathbb{Z})$ bundle on $\mathcal{C}$. It turns out that (in every known example) this $S p(2 r, \mathbb{Z})$ "periodicity" of $\tau_{a b}$ can be geometrically encoded by taking $\tau_{a b}$ to be the complex structure (period matrix) of a genus- $r$ Riemann surface $\Sigma$. For the models I will discuss $\Sigma$ is hyperelliptic and so can be presented as a two-sheeted cover of the complex $x$ plane described by the complex curve $y^{2}=P(x)$ where $P(x)$ is a degree $2 r+1$ or $2 r+2$ polynomial.

The curve for the scale invariant $S p(2 r)$ theory with $2 r+2$ fundamental quarks is [7]

$$
x y^{2}=\left[x \prod_{a=1}^{r}\left(x-\phi_{a}^{2}\right)+g \prod_{j=1}^{2 r+2} m_{j}\right]^{2}-g^{2} \prod_{j=1}^{2 r+2}\left(x-m_{j}^{2}\right) .
$$

(Note that the polynomial on the right side is divisible by $x$.) Here the quark masses $m_{j}$ transform in the adjoint of the $S O(4 r+4)$ flavor group. The $\phi_{a}$ can be taken as the eigenvalues of the Higgs field $\langle\phi\rangle=\operatorname{diag}\left\{\phi_{1}, \ldots, \phi_{r}\right.$, $\left.-\phi_{1}, \ldots,-\phi_{r}\right\}$. A basis of gauge invariant combinations of the $\phi_{a}$ 's are the symmetric polynomials in $\phi_{a}^{2}$ up to degree $2 r . g$ is a function of the coupling which must go as $8 e^{i \pi \tau}$ at weak coupling. In the scale invariant theory (setting the masses to zero) the coupling parameter space is thus the $g^{2}$ plane; weak coupling is at $g^{2}=0$, ultra-strong coupling corresponds to the $g^{2}=-1$ singularity, while the $\mathbb{Z}_{2}$ orbifold point is at $g^{2}=\infty$, since it is a fixed point of the $g \rightarrow-g$ symmetry of the scale invariant curve. This shows how the low-energy effective action encodes the S-duality by making its parameter space isomorphic to a fundamental domain of $\Gamma_{0}(2)$. This has been the main evidence for the existence of this duality to date.

The Coulomb branch of the scale invariant $S U(r+1)$ theory with $2 r+2$ fundamental quarks is described by the curve [6]

$$
y^{2}=\prod_{a=1}^{r+1}\left(x-\phi_{a}\right)^{2}+\left(h^{2}-1\right) \prod_{j=1}^{2 r+2}\left(x-\mu_{j}-h \mu\right) .
$$

Here the quark masses $\mu_{j}$ transform in the adjoint of the $S U(2 r+2)$ flavor group and satisfy the tracelessness constraint $\sum_{j} \mu_{j}=0$, while the singlet mass $\mu$ is charged under the $U(1)$ flavor group ("baryon number"). The $\phi_{a}$ can be taken as the eigenvalues of the Higgs field $\langle\phi\rangle=\operatorname{diag}\left\{\phi_{1}, \ldots, \phi_{r+1}\right\}$, subject to the constraint $\sum_{a} \phi_{a}=0$. The gauge invariant combinations of 
the $\phi_{a}$ 's are all the symmetric polynomials in $\phi_{a}$ up to degree $r+1$. $h$ is a function of the coupling such that $h \sim 1+32 e^{i \pi \tau}$ at weak coupling. In the scale invariant theory (setting the masses to zero) the coupling parameter space is the $h^{2}$ plane; weak coupling is at $h^{2}=1$, ultra-strong coupling corresponds to the $h^{2}=0$ singularity, while the $\mathbb{Z}_{2}$ orbifold point is at $h^{2}=\infty$, much as in the $S p(2 r)$ case.

The curves for the scale invariant $S O(n)$ theories [7] are quite similar, and encode the S-duality group in the low-energy effective action in an identical fashion.

In order to derive these S-dualities as exact equivalences of the entire theory, we will need the effective action for related asymptotically free theories, obtained by starting from the above solutions and flowing down by turning on bare quark masses. In particular the curve for the asymptotically free $S p(2 r)$ theory with $2 r$ massless flavors is found by making $2 r$ masses in (3.1) vanish and setting the remaining two masses to $M \rightarrow \infty$ while keeping $\Lambda^{2}=8 e^{i \pi \tau} M^{2} \sim g M^{2}$ finite. $\Lambda$ is the strong coupling scale of the asymptotically free theory. (I have chosen the finite factor of proportionality in its definition for later convenience.) Thus the massless asymptotically free $S p(2 r)$ theory with $2 r$ flavors has a Coulomb branch described by the curve

$$
y^{2}=x \prod_{a=1}^{r}\left(x-\phi_{a}^{2}\right)^{2}-\Lambda^{4} x^{2 r-1} .
$$

Similarly, the asymptotically free $S U(r+1)$ curve with $2 r$ flavors is found by setting $2 r$ adjoint masses to $-M$, the other two to $r M$, the singlet mass to $M+\mu$, and taking the $M \rightarrow \infty$ limit while keeping $\Lambda^{2}=-64 e^{i \pi \tau} M^{2} \sim$ $\left(h^{2}-1\right) M^{2}$ finite. The resulting curve is

$$
y^{2}=\prod_{a=1}^{r+1}\left(x-\phi_{a}\right)^{2}-(r+1)^{2} \Lambda^{2}(x-\mu)^{2 r} .
$$

Here I have kept a finite singlet mass $\mu$ in the asymptotically free theory for later convenience.

\section{Deriving the S-duality of the $S p, S O$, and $S U$ Series}

We now have the necessary background in place to derive the conjectured $\mathrm{S}$-dualities of the scale invariant models. I will start with the $S p$ series. The idea is to take the asymptotically free $S p(2 r)$ theory with $2 r$ quarks as the enlarged theory in which a scale invariant $S p$ theory will be embedded. 
We can flow to a scale invariant $S p(2 r-2)$ theory by tuning the Higgs vevs $\phi_{a}$ so as to break $S p(2 r) \rightarrow S p(2 r-2) \times S U(2)$ while keeping the quarks massless in the $S p(2 r-2)$ factor. This is achieved classically by letting one of the $\phi_{a}$ be non-zero, say $\phi_{r}=M$, while setting the rest to zero. That this is also true quantumly can be seen by examining the curve (3.3), which should factorize into a piece corresponding to the singularity at the origin of the Coulomb branch of the $S p(2 r-2)$ factor (i.e. the scale invariant theory we are interested in) and a non-singular piece corresponding to the $S U(2)$ Yang-Mills factor. The scale invariant singularity at the origin of the Coulomb branch for $S p(2 r-2)$ is simply $y^{2}=x^{2 r-1}$ as can be seen by setting all the Higgs vevs and quark masses to zero in (3.1). Eq. (3.3) indeed factorizes in this way if we set $\phi_{r}=M$ and the rest to zero:

$$
y^{2}=x^{2 r-1}\left[\left(x-M^{2}\right)^{2}-\Lambda^{4}\right] .
$$

The one dimensionless parameter at our disposal in this tuning, namely $M^{2} / \Lambda^{2}$, must be identified with the one dimensionless parameter (the coupling) of the scale invariant $S p(2 r-2)$ theory. (One can make this identification more explicit by leaving in finite masses and Higgs vevs in the asymptotically free $S p(2 r)$ theory, and taking $\Lambda$ and $\phi_{r}=M$ to infinity keeping their ratio fixed. In the limit one directly obtains the scale invariant $S p$ curve (3.1) with $g=\Lambda^{2} / M^{2}$.)

We have thus derived the quantum coupling space of the scale invariant $S p$ theories as the complex $M^{2}$ plane. Note that $M^{2} \rightarrow \infty$ corresponds to weak coupling since then the asymptotically free enlarged theory breaks in the ultraviolet. There are also two special points at $M^{2}= \pm \Lambda^{2}$ where the term in square brackets in (4.1) has a zero. These we identify with new (ultra)strong coupling physics as they imply the scale invariant $S p(2 r-2)$ theory includes additional degrees of freedom at those couplings. In summary, the quantum coupling space of the scale invariant $S p$ theories seems to have one weak coupling and two ultra-strong coupling points. This does not match with the conjectured $\Gamma_{0}(2)$ S-duality of these theories which we saw above is equivalent to having a quantum coupling space with one weak coupling, one ultra-strong coupling and one $\mathbb{Z}_{2}$ orbifold point.

However, the asymptotically free theory has a global (non-anomalous) $\mathbb{Z}_{2}$ R-symmetry which acts on the $M^{2}$-plane as $M^{2} \rightarrow-M^{2}$ (i.e. it is spontaneously broken away from the origin of the Coulomb branch). The $M^{2}$ plane and hence the quantum coupling space of the scale invariant theory is to be divided by the action of this symmetry, which then gives one weak coupling point at infinity, one ultra-strong coupling point at $M^{4}=\Lambda^{4}$, and a $\mathbb{Z}_{2}$ orbifold point at the origin. We have thus derived the S-duality of the $S p$ series. 
(In the case of non-zero bare masses, the $\mathbb{Z}_{2}$ global symmetry used above is the one described in [9] for $S U(2)$. It is a subgroup of the non-anomalous $\mathbb{Z}_{8}$ found by an appropriate combination of a global $U(1)_{R}$, the $\mathbb{Z}_{2}$ parity of the $O(4 r)$ flavor group, and a global $S U(2)_{R}$ transformation. It acts on the Higgs and quark fields by $\phi_{a} \rightarrow e^{i \pi / 2} \phi_{a}, q_{1} \rightarrow e^{-i \pi / 4} \widetilde{q}_{1}, \widetilde{q}_{1} \rightarrow e^{-i \pi / 4} q_{1}$, $q_{j} \rightarrow e^{-i \pi / 4} q_{j}$, and $\widetilde{q}_{j} \rightarrow e^{-i \pi / 4} \widetilde{q}_{j}$.)

Very similar manipulations can be applied to the $S O$ series of models. By tuning Higgs vevs in an asymptotically free $S O(n)$ theory with $n-4$ massless flavors to give the scale invariant $S O(n-2)$ theory, one derives its conjectured S-duality group in essentially the same way.

The $S U$ series is slightly different because one must also tune a non-zero quark mass in the asymptotically free $S U(r+1)$ theory with $2 r$ quarks to find the scale invariant $S U(r)$ theory. Classically we achieve this breaking by tuning the Higgs vevs so that $\phi_{a}=M$ for $1 \leq a \leq r$ and $\phi_{r+1}=-r M$ (by the tracelessness condition). In addition we must tune the singlet mass to $\mu=M$ to keep the $2 r$ quarks massless. These tunings are also valid quantumly since upon applying them to the asymptotically free $S U$ curve (3.4), and shifting $x \rightarrow x+M$, it factorzes as

$$
y^{2}=x^{2 r}\left[(x+(r+1) M)^{2}-(r+1)^{2} \Lambda^{2}\right],
$$

and we recognize the $x^{2 r}$ factor as the singularity of the scale invariant $S U(r)$ theory with $2 r$ massless quarks. We must identify the dimensionless parameter $M / \Lambda$ which we have tuned with the coupling of the scale invariant theory; one can explicitly check from the form of the curves that $\Lambda^{2} / M^{2}=$ $1-h^{2}$. From the degenerations of (4.2) the quantum coupling space has a weak coupling point at $M=\infty$ and two ultra-strong coupling points at $M= \pm \Lambda$. Furthermore there is a non-anomalous $\mathbb{Z}_{2} \in U(1)_{R}$ which acts on the Higgs fields as $\phi \rightarrow-\phi$ (and when appropriately combined with a global flavor rotation takes $\mu \rightarrow-\mu$ ), so that the $M$ plane is identified under $M \rightarrow-M$, giving a single ultra-strong coupling point and a $\mathbb{Z}_{2}$ orbifold point at $M=0$, thus deriving the content of the conjectured S-duality for the $S U$ series.

\section{Deriving the $S L(2, \mathbb{Z})$ Duality of the $S U(2) \simeq$ $S p(2)$ Theory}

Though both the $S p$ and $S U$ series include the $S U(2) \simeq S p(2)$ theory with four fundamental quarks, their S-dualities derived above do not include the full $S L(2, \mathbb{Z})$ duality argued to hold in this theory [9]. For example, upon specializing the $S U$ scale invariant curve (3.2) to the $S U(2)$ case, one finds that the curve is invariant under the following redefinition of the coupling 
$h[6]:$

$$
U: h \rightarrow \frac{1-h}{1+3 h}
$$

(with an accompanying $S L(2, \mathbb{C})$ transformation on the $x$ coordinate). When combined with the $\widetilde{\Gamma}^{0}(2)$ duality derived above, this gives rise to the full $S L(2, \mathbb{Z})$ duality-namely, a quantum coupling space with a weak coupling point, a $\mathbb{Z}_{2}$ orbifold point, a $\mathbb{Z}_{3}$ orbifold point, and no ultra-strong coupling point. (This is easiest to see in the $h$ plane where there are weak coupling points at $h= \pm 1$ and an ultra-strong point at $h=0$. The $\widetilde{\Gamma}^{0}(2)$ duality is then generated by $V: h \rightarrow-h$. The combined group generated by $U$ and $V$ is isomorphic to $S L(2, \mathbb{Z})$.) It is clear, however, that the nonlinear transformation (5.1) cannot be realized as a global symmetry in the enlarged asymptotically free $S U(3)$ theory.

Nevertheless, we can deduce this nonlinear equivalence from a global symmetry in another way. Consider instead the $S U(2)$ theory as part of the $S p$ series. We derived the $\Gamma_{0}(2)$ duality of this model above, realizing the redefinition

$$
U^{\prime}: g \rightarrow-g
$$

of the coupling $g$ of the $S p(2)$ theory as a global symmetry of the asymptotically free $S p(4)$ theory. Now, the equivalence of the scale invariant $S U(2)$ and $S p(2)$ curves (3.2) and (3.1) implies that their coupling parameters must be related by

$$
h=\frac{g+1}{3 g-1} .
$$

(See [7] for details, especially section 3.4.) Combining (5.2) with (5.3) gives (5.1), and thus derives the full $S L(2, \mathbb{Z})$ duality of the $S U(2) \simeq S p(2)$ scale invariant theory. Thus, by enlarging the $S U(2)$ theory in two different ways, different generators of its S-duality group are realized as global symmetries which together generate the full group. This can be understood pictorially by noting that the intersection of the fundamental domains of $\Gamma_{0}(2)$ and $\widetilde{\Gamma}^{0}(2)$ in fig. 1 is a fundamental domain of $S L(2, \mathbb{Z})$.

It is a pleasure to thank A. Buchel and A. Shapere for many helpful discussions. This work was supported by NSF grant PHY-9513717.

\section{References}

[1] C. Montonen and D. Olive, Phys. Lett. B72 (1977) 117; E. Witten and D. Olive, Phys. Lett. B78 (1978) 97; H. Osborn, Phys. Lett. B83 (1979) 321. 
[2] A. Sen, Phys. Lett. B329 (1994) 217; S. Sethi, M. Stern, and E. Zaslow, Nucl. Phys. B457 (1995) 484; J. Gauntlett and J. Harvey, Nucl. Phys. B463 (1996) 287; F. Ferrari, Nucl. Phys. B501 (1997) 53; K. Lee and P. Yi, hep-th/9706023.

[3] C. Vafa and E. Witten, Nucl. Phys. B432 (1994) 3.

[4] O. Aharony, C. Sonnenschein, S. Yankielowicz, and S. Theisen, Nucl. Phys. B297 (1997) 177; M. Douglas, D. Lowe, and J. Schwarz, Phys. Lett. B394 (1997) 297.

[5] J. Harvey, G. Moore and A. Strominger, Phys. Rev. D52 (1995) 7161; M. Bershadsky, A. Johansen, V. Sadov, and C. Vafa, Nucl. Phys. B448 (1995) 166.

[6] P. Argyres, M. Plesser, and A. Shapere, Phys. Rev. Lett. 75 (1995) 1699.

[7] P. Argyres and A. Shapere, Nucl. Phys. B461 (1996) 437.

[8] E. Witten, Nucl.Phys. B500 (1997) 3; K. Lansteiner, E. Lopez, and D. Lowe, Nucl. Phys. B507 (1997) 197; A. Brandhuber, J. Sonnenschein, S. Theisen, and S. Yankielowicz, Nucl. Phys. B504 (1997) 175.

[9] N. Seiberg and E. Witten, Nucl. Phys. B431 (1994) 484.

[10] I. Jack and H. Osborn, Nucl. Phys. B343 (1990) 647; H. Sonoda, Nucl. Phys. B383 (1992) 173; Nucl. Phys. B394 (1993) 302; B. Dolan, Int. J. Mod. Phys. A10 (1995) 2439.

[11] P. Argyres, Nucl. Phys. B (Proc. Suppl.) 61A (1998) 149.

[12] N. Seiberg and E. Witten, Nucl. Phys. B462 (1994) 19. 